\title{
ALTIRISCHE REIMSPRÜCHE.
}

Die folgenden fast alle noch der altirischen Periode entstammenden Reimereien finden sich in der von Michael O'Clery geschriebenen Handschrift B. IV. 2 der Königlichen Akademie zu Dublin, die ich Archiv III, S. 302 kurz beschrieben habe.

(fo. 130a)

1 In rannán dogniat-som sáncán a n-íathoiǔ Íli, is form-sa dusliat-somh: for coin liter cech ndíbhe.

2 Íssiol fri hard, ní bec búaidh; slemoin fri garù caich ar n-úair; intí diroindi samlaid, bidh réidh riëm cech n-aimhréidh ${ }^{1}$ ).

3 Cíall do duine ocus cruth, mogénor dian suidhiughudh; madh an cruth álainn namá, is corrthoir gan tablanna. Duine teimhen co céill nglain, mogénor fristibhe gen; madh in roálainn cen céill, ní mad aicci gréin dia len. ${ }^{2}$ )

4 Diamad mé douéradh fuiriocc, robadh méth cech manuc, ${ }^{3}$ ) nobetis déra geiredh dar eneach gach caratt.

5 A duine trúaigh, dirsan duit! it olc[a] álcho4) do choirp: tan it sáithech notge[i]b úall, ${ }^{5}$ ) it toirsech ${ }^{8}$ ) trúag [tan] it goirt.

6 Is gnáth do neoch iar tóchall toidecht ina athbóthar: súaill inní as buide le bocht cen co n-apar acht athgort.

1) Zu lesen amraid, wie soraid aus so-réid enstanden. Vgl. den Spruch Brigittens im Codex Bernensis (Zeitschr. IV, S. 186). Gedicht.

2) Mit diesen Versen vergleiche das Zeitschrift VI, S. 267 abgedruckte

8) Statt manach, gleichsam Deminutiv in -uc, des Reimes wegen.

‘) 0'Clery setzt in unbetonten Silben gern 0 statt $a$.

๖) waill MS.

๑) toirsich MS. 
K. MEYER, ALTIRISCHE REIMSPRÜCHE.

7 Ni coïr indus berdar in coïdh:

ní rop cōra dech in rí isin tír ataat inbi.

Is coïr indus berdar in coïdh:

sellaidh [ind] inti nat ibh, iǔidh inti dian coür.

8 Condig ind anim for cói co roseara frisin crí co rodeglat immale nocha dernat bith gan ni.

9 Is mo rí, is mo $\mathrm{t}[\mathrm{h}]$ igerna dogní;

is ferr guth in gabair mairbh iná gairm in ghobhair bí.

10 Bidh Buccaiti ainm do mheic, bidh Minsech mad ingen d[e]it, bid Gobairsech 1) t' ainm fadéin.

11 Ni dot choiriucchadh, a Dé, trúagh an sainiughudh dogni: marbai in ri[g] foloing in slúag, aingi in-trúagh nát túartéit ní.

(fo. $130 \mathrm{~b}$ )

12 Téid mo menma ${ }^{2}$ ) don mír fil il-láimh rígh Temra tréin: madh maith lais, douéra im láim, mad ferr lais, doméla féin.

13 Dia mo ruire, Dia mo rí, Dia mo duine, Dia mo dia, ní nomsainmescca ó mac Dé dirairmescca mac Dé dia.

14 Domuin gach sétach is rí, dáigh fogni bréccach ${ }^{3}$ ) bith cé; ecmaing is dó nach terc ni, cech rí dia tecmoing serc Dé.

15 Eser ni re ndol ar fecht, do thúaro ni rob imt[h]erc, acht ma tresoide do nert, ni ba mesoitti t' imt[h]echt.

16 Treabar doberar do grés, is é a bés cia roféis: beiridh a buidhe dotmél ${ }^{4}$ ), forbeir a bfágoibh dia éis.

17 A Chathail mic Finngoine ${ }^{5}$ ), fó lim cacha erbara, ceat olca na hairchinnigh, it maithe na hērlamha. ${ }^{6}$ )

1) gobairsich MS.

2) $\mathrm{Zu}$ lesen menma-sa, wenn nicht Reim mit Temra beabsichtigt ist.

3) mbreccach oder inbreccach MS.

4) $\mathrm{Zu}$ lesen dodmeil?

5) Besser Finguine.

6) Vgl. Ériu IV, S. 40, Anm.3.

Liverpool. Kuno Meyer. 